\title{
Locking bandwidth equations for electrically and optically injection locked oscillators
}

\author{
X. Wang, N. J. Gomes
}

\begin{abstract}
The two most widely used locking bandwidth equations for injection locked oscillators, Adler's and Kurokawa's equations, are compared for the first time. Three differences are pointed out from the comparison: first, the absence of the factor $1 / \cos \theta$ in Adler's equation; secondly, larger bandwidth is predicted by Kurokawa's equation; and thirdly, a difference in the $Q$ factors used. It is shown that Kurokawa's locking bandwidth equation is suitable for high- $Q$ reflection-type electrically injection-locked oscillators employing a circulator, while Adler's equation is suitable for reflection-type injection-locked oscillators not employing a circulator. Experimental results from measurements on an optically injection-locked oscillator show that the loaded- $Q$ varies with injection power, and demonstrate that both Kurokawa's and Adler's equations, which treat the $Q$ as a constant, are not accurate for predicting the locking bandwidth of wide locking bandwidth, injection-locked oscillators. Finally, a theoretical analysis of the optimum photodetector insertion point for a wide locking bandwidth, transmission-type indirect optically injection-locked oscillator is presented.
\end{abstract}

\section{Introduction}

The injection-locking of an electrical oscillator was first described by Van Der Pol in 1927 [1], and the first locking bandwidth equation for electrically injection-locked 
oscillators was developed by Adler in 1946 [2]. A vacuum tube transistor was used in Adler's oscillator model. The most comprehensive theoretical review for the injectionlocking of solid-state injection-locked oscillators was given by Kurokawa in 1973 [3]. The locking bandwidth equation of such oscillators was also derived in Kurokawa's paper. Compared with Adler's oscillator model, an additional circulator at the oscillator output in Kurokawa's model was used to separate the injection signal and the oscillator output signal. Adler's and Kurokawa's equations have been used widely to calculate the locking bandwidth for injection-locked oscillators [4, 5]. Kurokawa's theory was also used to develop a locking bandwidth equation for transmission type injection-locked oscillators [6]. Further, equations for such electrically injection-locked oscillators have been used for predicting the locking bandwidth of optically injection-locked oscillators [4]-[5]. In recent years, optically injection-locked oscillators have attracted great interest because of their possible use in future optical communication systems, in applications ranging from microwave and millimetre-wave signal reception, phased array systems and optoelectronic clock recovery [7]-[12]. Locking bandwidth is one of the major design parameters in these types of injection-locked oscillator.

In this paper, Kurokawa's and Adler's locking bandwidth equations are first compared. The models, assumptions, and analyses used by Kurokawa and Adler are described. Then the differences between the two equations, the factor $1 / \cos \theta$, the calculated locking bandwidth, and the Q factor are identified. The causes for these differences are discussed in detail.

Results from an experiment designed to investigate the dependence of oscillator loaded Q on the injection signal power using an optically injection-locked oscillator are then presented. One of the limitations of the locking bandwidth equations, the constant value of $\mathrm{Q}$ assumed, was investigated based on these results.

Transmission type indirect optically injection-locked oscillators potentially offer higher locking bandwidth than other types of optically injection-locked oscillator [7]. An optimum photodiode insertion point design is required by this type of oscillator for a 
wide locking bandwidth. A theoretical analysis for the optimum photodiode insertion point is presented at the end of this paper.

\section{Kurokawa's locking bandwidth equation}

A simple series resonant model of the microwave resonator was introduced by Kurokawa [3] to model the reflection type injection-locked oscillator. This model, shown in Fig 1, is based on the assumptions of reflection type injection-locking and small injection signal power compared with the oscillator free running output power. The oscillator active device is modelled as a negative impedance $\left(Z_{d}(A)\right)$. A series LC resonator is used to model the resonator. The resonator resistance is represented as $R_{a}$. The injection signal is applied to the oscillator output (a-a') through a circulator. The oscillator output voltage and current at the injection point are $V$ and $I$ respectively. The injection signal voltage is $E_{i}$, and the load resistance is $R_{L}$. In order to match the output, Kurokawa supposed the injection source resistance was $R_{L}$ as well. The quality factor of a resonator is very high $(>1000)$ for conventional oscillators, which means the resonator resistance $R_{a}$ is very small compared to the load resistance. So, Kurokawa ignored the injection power loss within the resonator. Based on the assumptions above, Kurokawa calculated the injection signal current to be $E_{i} / 2 R_{L}$, see Fig 1 . However, this assumption is not exactly correct for injection-locked oscillators when the resonator quality factor is quite low. The resonator circuit impedance as a function of frequency is written as:

$$
Z_{c}(\omega)=j\left(\omega L-\frac{1}{\omega C}\right)+R_{a}+R_{L}
$$

Assuming the free-running frequency is approximately the same as the resonance frequency of the resonator $\omega_{r}$, a Taylor expansion of (1) is performed to give:

$$
Z_{c}(\omega) \approx 2 j\left(\omega-\omega_{r}\right) L+R_{a}+R_{L}
$$


where:

$$
\omega_{r}=\frac{1}{\sqrt{L C}}
$$

and $\omega$ is the instantaneous frequency

The locking bandwidth is calculated using the impedance graph [3]. Here $\omega_{0}$ is the oscillator free-running frequency, the locking bandwidth of the oscillator $B$ is equal to $2 \Delta \omega_{m}, E_{i}$ is the injection signal amplitude, $\theta$ is the angle between the resonator impedance locus and the device line, and $A_{0}$ is the RF current amplitude. The distance between $\omega_{0}+\Delta \omega_{m}$ and $\omega_{0}-\Delta \omega_{m}$ on the resonator locus is calculated using (2),

$$
\begin{aligned}
& \left|Z_{c}\left(\omega_{0}+\Delta \omega_{m}\right)-Z_{c}\left(\omega_{0}-\Delta \omega_{m}\right)\right| \\
& =j\left[\left(\omega_{0}+\Delta \omega_{m}\right) L-\frac{1}{\left(\varpi_{0}+\Delta \omega_{m}\right) C}\right]+R_{a}+R_{L}-\left[j\left(\left(\omega_{0}-\Delta \omega_{m}\right) L-\frac{1}{\left(\omega_{0}-\Delta \omega_{m}\right) C}\right)+R_{a}+R_{L}\right] \\
& =2 j \Delta \omega_{m} L
\end{aligned}
$$

The relation between the injection vector and the locking bandwidth at the maximum locking frequency as shown in Fig 2 is,

$$
\left|2 \Delta \omega_{m} L \cos \theta\right|=\frac{\left|E_{i}\right|}{A_{0}}
$$

The oscillator free-running output power (the power that a free-running oscillator delivers to the load) can be calculated as:

$$
P_{0}=\frac{1}{2} R_{L} A_{0}^{2},
$$

such that,

$$
A_{0}=\sqrt{\frac{2 P_{0}}{R_{L}}}
$$


where $R_{L}$ is the load resistance. The injection signal power at the point of injection is,

$$
P_{i}=I_{i}^{2} R_{L}=\left(\frac{E_{i}}{2 \sqrt{2} R_{L}}\right)^{2} \cdot R_{L},
$$

such that,

$$
\left|E_{i}\right|=2 \sqrt{2} \cdot \sqrt{P_{i}} \cdot \sqrt{R_{L}}
$$

The external quality of the resonator is calculated by [3],

$$
Q_{\text {ext }}=\frac{\omega_{0} L}{R_{L}} \approx \frac{\omega_{r} L}{R_{L}}
$$

giving,

$$
\frac{R_{L}}{L}=\frac{\omega_{0}}{Q_{e x t}}
$$

Substituting (5), (6), and (7) into (4), the locking bandwidth equation can be written as:

$$
B=2 \Delta \omega_{m}=2 \cdot \frac{1}{\cos \theta} \cdot \frac{R_{L}}{L} \cdot \sqrt{\frac{P_{i}}{P_{0}}}=\frac{2 \omega_{0}}{Q_{e x t}} \sqrt{\frac{P_{i}}{P_{0}}} \frac{1}{\cos \theta}
$$

From (8), it can be seen that when $\theta=\pi / 2$, the resonator locus overlays the device line, and $B$ is infinite. When $\theta=0$, the resonator locus is perpendicular to the device line, and the locking bandwidth reaches the minimum value:

$$
B_{\min }=\frac{2 \omega_{0}}{Q_{e x t}} \sqrt{\frac{P_{i}}{P_{0}}}
$$

Normally, (9) is used for the theoretical prediction of the oscillator locking bandwidth because the angle between the resonator locus and the device line is difficult to calculate. 
The resonator loss does not affect the locking bandwidth in Kurokawa's equation. $Q_{e x t}$ in Kurokawa's equation is treated as a constant.

\section{Adler's locking bandwidth equation}

Fig 3 shows the circuit model used by Adler [2]. A parallel $R L C$ resonator was used for the resonator model, where $R$ is a resistance, giving the resonator loss. A tube transistor was used as the active device of the oscillator. $E_{0}$ is the free-running output voltage amplitude, $E_{i}$ is the injection signal voltage amplitude, and $E_{g}$ is the amplitude of the total voltage generated across the load after the application of the external signal.

A vector diagram of the signals within an injection-locked oscillator was presented by Adler, see Fig. 4 [2]. The oscillator instantaneous output is represented by the vector $E$ rotating clockwise with an angular frequency of $\omega$ (the instantaneous output frequency after injection locking) while the injection signal vector of amplitude, $E_{i}$, rotates clockwise with an angular frequency of $\omega_{i}$ (the injection signal frequency). The instantaneous phase difference between these vectors is $\alpha$. The phase difference between the instantaneous output frequency and the total voltage (of amplitude $E_{g}$ ) generated across the load, $\phi$, was given as [2],

$$
\phi=-\frac{E_{i}}{E} \sin \alpha
$$

$\phi$ can also be written as [2],

$$
\phi=\frac{d \phi}{d \omega}\left(\omega-\omega_{0}\right)=\frac{d \phi}{d \omega}\left[\left(\omega-\omega_{i}\right)-\left(\omega_{0}-\omega_{i}\right)\right]=\frac{d \phi}{d \omega}\left[\Delta \omega-\Delta \omega_{0}\right]
$$

where $\omega_{0}$ is the oscillator free-running output frequency, $\Delta \omega=\frac{d \alpha}{d t}=\omega-\omega_{i}$ is the instantaneous beat frequency, and $\Delta \omega_{0}=\omega_{0}-\omega_{i}$ is the undisturbed beat frequency. 
Substituting (11) into (10), we have [2],

$$
-\frac{E_{i}}{E} \sin \alpha=\frac{d \phi}{d \omega}\left[\frac{d \alpha}{d t}-\Delta \omega_{0}\right]
$$

For a single tuned resonator, the quality factor is [2]:

$$
Q=0.5 \omega_{0} \frac{d \phi}{d \omega}
$$

Substituting (13) into (12), we have [2],

$$
-\frac{E_{i}}{E} \sin \alpha=\frac{2 Q}{\omega_{0}}\left[\frac{d \alpha}{d t}-\Delta \omega_{0}\right]
$$

where $d \alpha / d t$ must be zero when the injection locked oscillator reaches a stable state. So in the steady state, (14) becomes [2]

$$
\frac{E_{i}}{E} \sin \alpha=\frac{2 Q}{\omega_{0}} \cdot \Delta \omega_{0},
$$

giving,

$$
B=2 \Delta \omega_{0}=\frac{\omega_{0}}{Q} \cdot \frac{E_{i}}{E}=\frac{\omega_{0}}{Q} \sqrt{\frac{P_{i}}{P_{0}}}
$$

\section{Differences between Adler's and Kurokawa's locking bandwidth equation}

There are three differences between Alder's locking bandwidth equation (15) and Kurokawa's locking bandwidth equation (8).

1. The absence of $1 / \cos \theta$ in Adler's equation. 
The reason for this is that Adler's equation corresponds to the minimum locking bandwidth case (Kurokawa's equation (9)), and is only suitable for predicting the minimum locking bandwidth $(\theta=0)$.

2. Larger bandwidth is predicted by Kurokawa's equation

Under the same conditions, the locking bandwidth predicted by Kurokawa is two times larger than Adler's prediction. To find the reason for this difference, we need to investigate the models that Kurokawa and Adler used to derive their locking bandwidth equations. As a circulator is included in Kurokawa's model (Fig. 1), the output power is dissipated only at the load, and the external quality factor is given as,

$$
Q_{e x t}=\frac{\omega_{0} L}{R_{L}}
$$

With no such circulator assumed in Adler's model (Fig. 3), the output power is dissipated in both the injection source resistor and the load in his analysis.

If the circulator is omitted from Kurokawa's model, the output power will be dissipated at both the load and source resistor, and the injection source resistor should be included for calculation of the oscillator's external quality factor:

$$
Q_{\text {ext }}=\frac{\omega_{0} L}{2 R_{L}}
$$

Substituting (17) into (8), we have:

$$
B=2 \Delta \omega_{m}=2 \cdot \frac{1}{\cos \theta} \cdot \frac{R_{L}}{L} \cdot \sqrt{\frac{P_{i}}{P_{0}}}=\frac{\omega_{0}}{Q_{\text {ext }}} \sqrt{\frac{P_{i}}{P_{0}}} \frac{1}{\cos \theta}
$$




$$
B_{\min }=\frac{\omega_{0}}{Q_{e x t}} \cdot \sqrt{\frac{P_{i}}{P_{0}}}
$$

We can conclude that (19) is identical to Adler's locking equation (15) except for the difference in $\mathrm{Q}$ factor which is discussed below.

3. The $Q$ factor.

In Adler's equation the $Q$ is the resonator loaded $Q$ instead of the external $Q$. Looking at Kurokawa's model in Fig 1, the injection signal current generated by the injection source is injected into the oscillator output via a circulator. This injection signal flows through the active device and the resonator, and is mixed with the oscillator free-running signal. The injection-locked oscillator free-running output signal containing the injection signal is applied to the load through the circulator. All resistors within this injection loop will cause a voltage drop and should be included in the calculation. These resistors include the injection source resistance (assumed to be $R_{L}$ ), the load resistance $R_{L}$, and the resonator resistance $R_{a}$. We can rewrite the equations in terms of the injection power $P_{i}$,

$$
P_{i}=\frac{1}{2} \cdot\left(\frac{E_{i}}{2 R_{L}+R_{a}}\right)^{2} \cdot R_{L},\left|E_{i}\right|=\frac{\sqrt{2} \cdot \sqrt{P_{i}} \cdot\left|2 R_{L}+R_{a}\right|}{\sqrt{R_{L}}}
$$

The loaded Q of the resonator is:

$$
Q=\frac{\omega_{0} L}{R_{L}+R_{a}}, \frac{R_{L}+R_{a}}{L}=\frac{\omega_{0}}{Q}
$$

Substituting (5) and (20) into (4), we obtain:

$$
\left|2 \Delta \omega_{m} L \cos \theta\right|=\frac{\sqrt{P_{i}} \cdot\left|2 R_{L}+R_{a}\right|}{\sqrt{P_{0}}}
$$




$$
\Delta \omega_{m}=\frac{1}{2} \cdot \frac{1}{\cos \theta} \cdot \frac{\sqrt{P_{i}}}{\sqrt{P_{0}}} \cdot\left(\frac{R_{L}+R_{a}}{L}+\frac{R_{L}}{L}\right)
$$

Substituting (21) and (7) into (23), we have:

$$
\Delta \omega_{m}=\frac{1}{2} \cdot \frac{1}{\cos \theta} \sqrt{\frac{P_{i}}{P_{0}}} \cdot\left(\frac{\omega_{0}}{Q}+\frac{\omega_{0}}{Q_{e x t}}\right)
$$

The locking bandwidth $\mathrm{B}$ is:

$$
B=2 \Delta \omega_{m}=\frac{1}{\cos \theta} \sqrt{\frac{P_{i}}{P_{0}}} \cdot\left(\frac{\omega_{0}}{Q}+\frac{\omega_{0}}{Q_{\text {ext }}}\right)
$$

The omission of $R_{a}$ in Kurokawa's calculation is accurate for conventional oscillators (high Q), as $R_{a}$ is small compared with $R_{L}$. However, for wideband injection locked oscillators, where $R_{a}$ is designed to be large and cannot be ignored, (25) should be used to calculate the correct locking bandwidth.

When the circulator is omitted, the output signal is dissipated at the injection source as well as the load, and the oscillator loaded Q can be rewritten as:

$$
Q=\frac{\omega_{0} L}{2 R_{L}+R_{a}}, \frac{2 R_{L}+R_{a}}{L}=\frac{\omega_{0}}{Q}
$$

Substituting (26) in to (23), we have

$$
\Delta \omega_{m}=\frac{1}{2} \cdot \frac{1}{\cos \theta} \cdot \frac{\omega_{0}}{Q} \sqrt{\frac{P_{i}}{P_{0}}}
$$

and the minimum locking bandwidth is then:

$$
B=2 \Delta \omega_{m}=\frac{\omega_{0}}{Q} \cdot \sqrt{\frac{P_{i}}{P_{0}}}
$$

This equation is identical to Adler's locking bandwidth equation (15). Adler's equation is thus correct for predicting the minimum locking bandwidth of reflection type injectionlocked oscillators without a circulator. 


\section{Dependence of oscillator loaded $Q$ on the injection signal power}

A millimetre-wave transmission-type optically injection-locked oscillator [7] was used to investigate the dependence of the oscillator loaded $\mathrm{Q}$ on the injection signal power.

The loaded Q for the reflection-type injection-locked oscillator was measured using the experimental setup shown in Fig.5. The oscillator was locked electrically by the injection-source. The injection signal was applied through a $25.6-40 \mathrm{GHz}$ circulator from Midisco (Isolation: $14 \mathrm{~dB}$, Insertion loss: $0.8 \mathrm{~dB}$ ). The oscillator output was connected with one of the circulator ports, and the injection signal from an HP 83640L $10 \mathrm{MHz}-40 \mathrm{GHz}$ signal source was injected into the oscillator through the circulator. The locking bandwidth was measured by an electrical spectrum analyser (HP8563E).

As the resonator loss is small, the difference between loaded Q and external Q is small, $Q \approx Q_{\text {ext }}$ and, therefore, (9) is used to calculate the oscillator loaded $\mathrm{Q}$.

Table 1 shows the calculated loaded Q. The locking bandwidth increased with injection power. The loaded Q of the oscillator varies from 240 to 550 depending on the injection signal power. The higher the injection signal power, the lower the loaded Q. The reason for this is that the oscillator $\mathrm{Q}$ is also dependent on the active device (HEMT) characteristics and these change with signal power.

\section{Photodiode insertion point}

For wide locking bandwidth transmission type indirect optically injection-locked oscillators, the photodiode insertion point needs to be optimised for maximum injection signal power.

For the analysis, see Fig 6, it is assumed that one end of the resonator is short-circuited, and the other is connected with the active device of the oscillator. $R_{L}$ is used to model 
loading effect of the active device. The power delivered to $R_{L}$ needs to be maximised for wide locking bandwidth (maximum current flow through $R_{L}$ ). The characteristic impedance of the resonator is $Z_{l}$. The photodiode is modelled by a current source, $i_{p}$. Photodiode capacitance and resistance are ignored for simplicity. The photodiode is inserted at a distance $x$ from the RF short. The distance between the insertion point and the load is $y . Z_{\text {input }}$ is the impedance looking from the insertion point to the RF short, and $Z_{\text {output }}$ is the input impedance looking from the insertion point to the load. The output current $i_{\text {output }}$, is:

$$
i_{\text {output }}=i_{p} \cdot \frac{Z_{\text {input }}}{Z_{\text {output }}+Z_{\text {input }}}
$$

where

$$
\begin{aligned}
& Z_{\text {input }}=j Z_{1} \tan (\beta x) \\
& Z_{\text {output }}=Z_{1} \frac{R_{L}+j Z_{1} \tan (\beta y)}{Z_{1}+j \cdot R_{L} \cdot \tan (\beta y)}
\end{aligned}
$$

and

$$
\beta=\frac{2 \pi}{\lambda_{g}}
$$

$\lambda_{\mathrm{g}}$ is the wavelength in the transmission line guide at the design frequency. Consider both $Z_{\text {input }}$ and $Z_{\text {output }}$ are positive; then it is not difficult to conclude from (31), that $Z_{\text {output }}$ should be as small as possible to maximise $i_{\text {output }}$. Minimum $Z_{\text {output }},\left(=R_{L}\right)$, is obtained when $y=0$ in (30); then (30) becomes,

$$
i_{\text {output }}=i_{p} \cdot \frac{Z_{\text {input }}}{R_{L}+Z_{\text {input }}}
$$

If we fix the length $x$ to a quarter-wavelength ( $\beta x=\pi / 4$ ), $Z_{\text {input }}$ will be infinity, and $R_{L}$ in the denominator of (34) can be ignored, and the maximum output current, $i_{\text {output }}=i_{p}$ is 
obtained. Therefore, to maximise the output current, the resonator length should be a quarter-wavelength and the photodiode should be inserted at the load end of the resonator. Another explanation of this quarter wavelength configuration resonator is that the quarter-wavelength resonator transforms the RF short circuit to open circuit. The photocurrent from the photodiode that is inserted at the end of the resonator will flow to the load instead of to the high-impedance RF open circuit.

In the real oscillator design where the photodiode capacitance can not be ignored, the photodiode capacitance can be matched by the bondwire inductance.

\section{Conclusion}

Three differences between Kurokawa's and Adler's locking bandwidth equations have been presented. These are:

1) The factor $1 / \cos \theta$.

2) The calculated locking bandwidth

3) Q factor.

It has been shown that Kurokawa's equation is only applicable to high Q reflection type electrical injection-locked oscillators that employ circulators. Even though Adler's locking equation is developed for reflection type electrical injection-locked oscillators, his equation is not suitable for such oscillators, as the circulator required for these types of oscillator to isolate the injection and oscillator free-running signals is not included in his model. However, his equation is a good theoretical basis for developing the locking bandwidth equation for reflection type injection-locked oscillators which do not require circulators, such as reflection type optically injection-locked oscillators.

It has been demonstrated that the loaded Q of an optically injection-locked oscillator changes with the injection signal power. Both Adler's and Kurokawa's equations treat the $\mathrm{Q}$ as a constant. New locking bandwidth analyses including the effect of changing Q 
with injection power need to be developed for predicting the locking bandwidth with good accuracy.

The analysis of optimum photodiode insertion point for a transmission type indirect optically injection-locked oscillator employing a short-circuited resonator has been presented. For a wide locking bandwidth design the injection signal power must be maximised, and it has been shown that the resonator length should be a quarterwavelength and the photodiode should be inserted at the load end of the resonator.

\section{Acknowledgements}

The authors are grateful to David Wake, then of BT Laboratories, now of Microwave Photonics Inc. for the provision of high-speed photodiodes used in the fabrication of millimetre-wave optically injection-locked oscillators. They also acknowledge the UK Engineering and Physical Sciences Research Council for supporting the project "Broadband Integrated Millimetre-Wave Over Fibre Systems", part of the Optoelectronic Systems Integration programme, during the period 1999-2001, when most of the experimental work for this paper was carried out. 


\section{References}

[1] B. Van Der Pol, "Forced oscillator in a circuit with nonlinear resistance," Phil. Mag., pp. 65-80, Vol. 3, 1927.

[2] R. Adler, "A study of locking phenomena in oscillators," Proceedings of the I.R.E. and Waves and Electrons, pp. 351-357, 1946.

[3] K. Kurokawa, "Injection locking of microwave solid-state oscillators," Proceedings of the IEEE, Vol.61, No.10, pp. 1386-1410, 1973.

[4] T. Tokumitsu, K. Kamogawa, I. Toyoda, and M. Aikawa, "A novel injection-locked oscillator MMIC with combined ultrawide-band active combiner/divider and amplifiers," IEEE Trans. Microwave Theory and Tech., Vol. 42, No. 12, pp. 2572-2578, 1994.

[5] D. Sommer and N. J. Gomes, "Wide-locking bandwidth optically injection-locked oscillators: Sparameter design and modulation effects," IEEE Transactions on Microwave Theory and Tech.., Vol. 43, pp. 1424-1434, 1995

[6] Y. Tajima and K. Mishima, "Transmission-type injection locking of GaAs schottky-barrier FET oscillators," IEEE Trans. on Microwave Theory and Tech., Vol. MTT-27, No.5, pp. 386-391, 1979.

[7] X. Wang, N. J. Gomes, L. Gomez-Rojas, P. A. Davies, and D. Wake, "Indirect optically injectionlocked oscillator for millimeter-wave communication system," IEEE Trans. Microwave Theory Tech., Vol 48, No. 12, pp. 2596-2603, 2000.

[8] H. Kamitsuna, T. Shibata, K. Kurishima,and M. Ida, "Direct optical injection locking of InP/InGaAs HPT oscillator ICs for Microwave Photonics and 40-Gbit/s-class optoelectronic clock recovery," IEEE Trans. Microwave and Tech., Vol. 50, No. 12, pp. 3002-3008, 2002.

[9] H. Ogawa and D. Polifko, "Fibre Optic Millimetre-wave subcarrier transmission links for personal radio communication systems," IEEE MTT-S Int. Dig., pp. 555-558, 1992.

[10] H. Ogawa, D. Polifko, and S. Banba, "Millimetre-wave fibre optics systems for personal radio communication," IEEE Trans. Microwave Theory and Tech., Vol. 40, No.12, pp. 2285-2293, 1992.

[11] D. Wake , L. D. Westbrook, N. G. Walker and I. C. Smith, "Microwave and millimetre-wave radio fibre," BT Technical J., Vol. 11, No. 2, pp. 76-88, 1993.

[12] X. Wang, "Millimetre-Wave Optically Injection-Locked Oscillators for Radio-Over-Fibre Systems," PhD thesis, submitted to the University of Kent, Canterbury, 2004. 


\section{LIST OF FIGURE CAPTIONS}

Fig 1 The equivalent circuit of an injection-locked oscillator with a single tuned resonator as presented by Kurokawa [3].

Fig 2 Calculation of the locking bandwidth as presented by Kurokawa [3].

Fig 3 Injection-locked oscillator circuit as presented by Adler [2]

Fig 4 Vector diagram of the injection-locked oscillator as presented by Adler [2].

Fig 5 Measurement of the loaded Q of a transmission-type optically injection-locked oscillator

Fig 6 Circuit model for a short-circuited resonator with photodiode.

\section{TABLE CAPTION}

Table 1 Loaded Q of the optically injection-locked oscillator. 


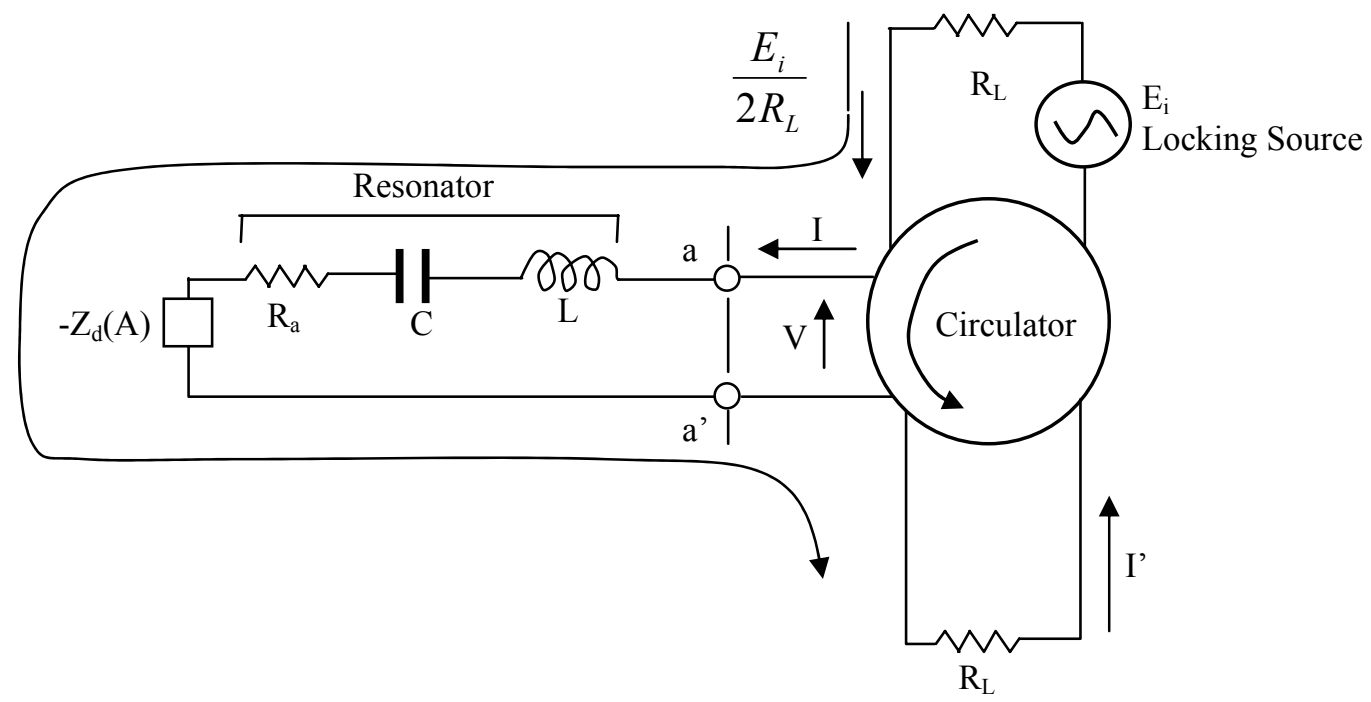




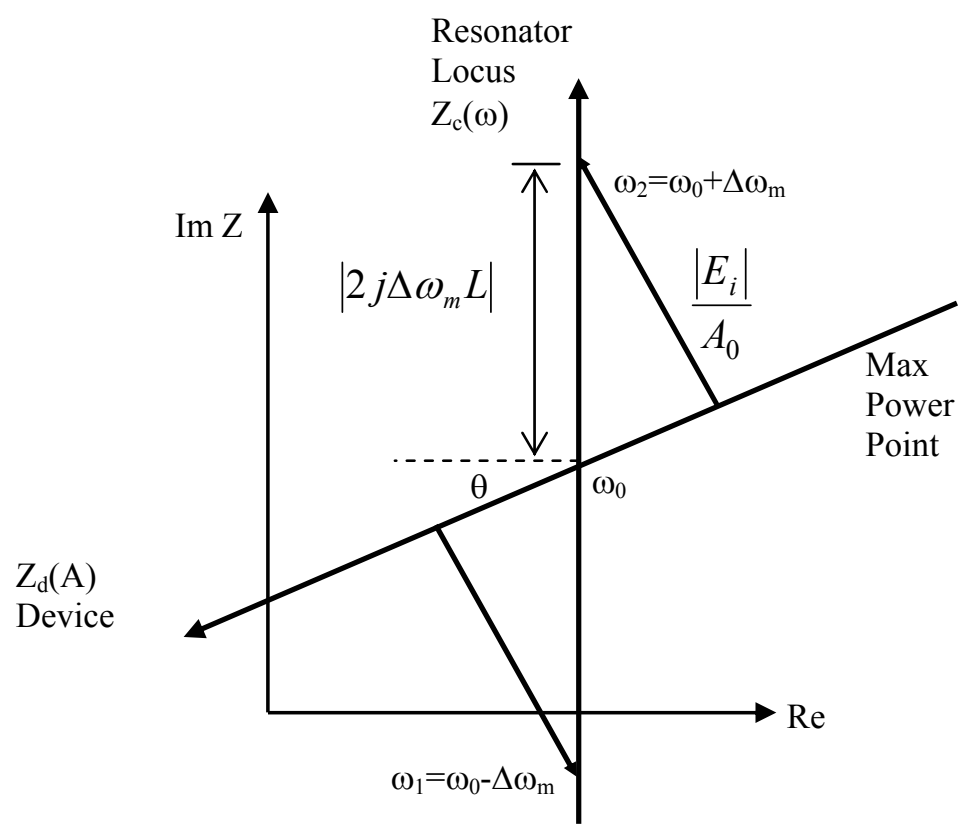




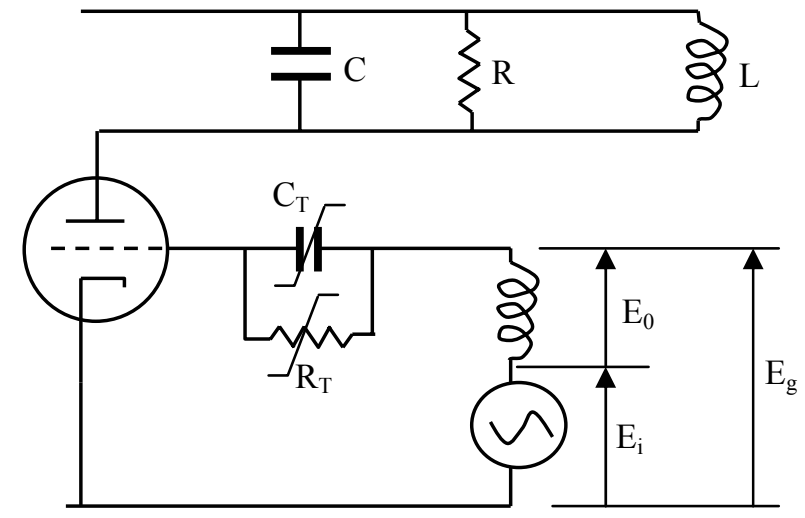




$$
\mathbb{Z}^{2}
$$




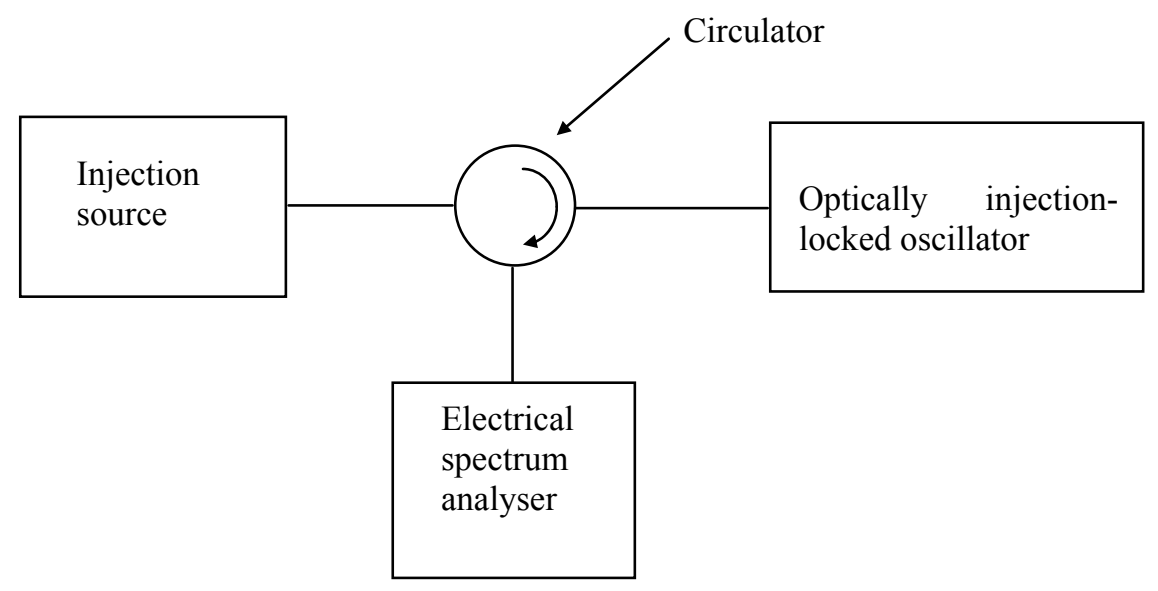




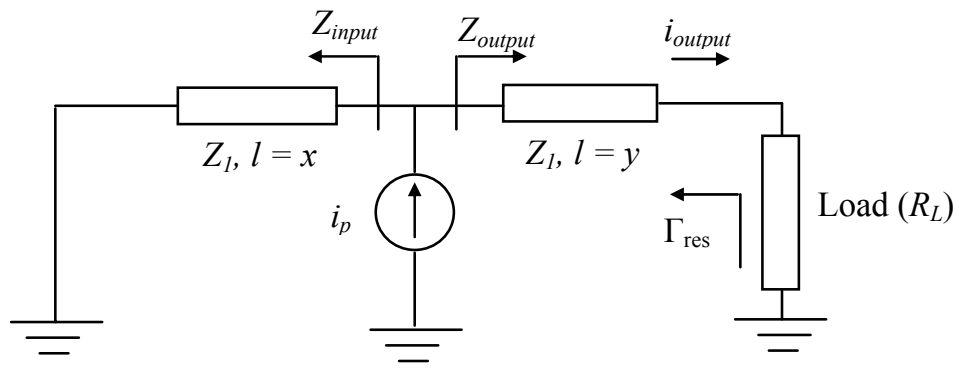




\begin{tabular}{|l|l|l|l|l|}
\hline $\begin{array}{l}\text { Free running } \\
\text { frequency }(\mathrm{Hz})\end{array}$ & $\begin{array}{l}\text { Locking } \\
\text { bandwidth }(\mathrm{Hz})\end{array}$ & $\begin{array}{l}\text { Injection signal } \\
\text { power }(\mathrm{dBm})\end{array}$ & $\begin{array}{l}\text { Free running signal } \\
\text { power }(\mathrm{dBm})\end{array}$ & Loaded Q \\
\hline $3.13 \mathrm{E}+10$ & $3.80 \mathrm{E}+06$ & -26.5 & -3 & 550.1525721 \\
\hline $3.13 \mathrm{E}+10$ & $2.76 \mathrm{E}+07$ & -16.5 & -3 & 239.6819092 \\
\hline $3.13 \mathrm{E}+10$ & $7.57 \mathrm{E}+07$ & -6.5 & -3 & 276.3429937 \\
\hline
\end{tabular}

